Beobachtungen des Kometen 1903 IV (1903 c).

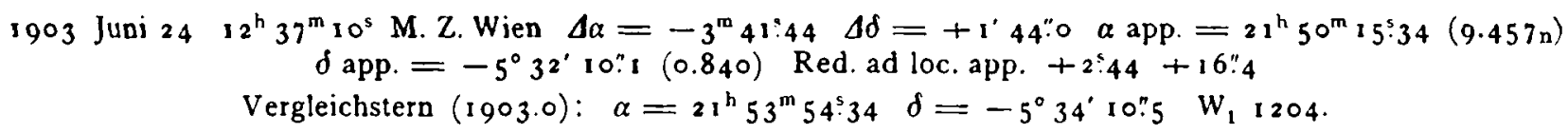

In der folgenden Zusammenstellung ist $h_{1}$ die Hellig. baren Schweifansatz auffallender, ohne denselben aber etwas keit des im 6 zöll. Refraktor erkennbaren Kerns, $h_{3}$ die schwächer als $\delta$ Ursae maj. ( $\left.3^{\mathrm{m}} \cdot 5\right)$. - Am II. August war Helligkeit der Kerngegend bis zu ' 'Durchmesser, $D$ der er so hell wie $\chi$ Ursae maj. ( $\left.3^{\mathrm{m}} \cdot 9\right)$, am 14 . wie $\nu$ Ursae Durchmesser des ganzen Kopfes des Kometen, $C$ die im maj. ( $3^{\mathrm{m}} \cdot \mathrm{f}$ ).

Refraktor eventuell auch im Sucherfernrohr gesehene Schweif. länge und $H$ die beobachtete Gesamthelligkeit.

\begin{tabular}{|c|c|c|c|c|c|c|c|}
\hline \multicolumn{2}{|c|}{1903} & $\begin{array}{l}\text { M. Z. } \\
\text { Wien }\end{array}$ & $h_{1}$ & $h_{2}$ & $D$ & $C$ & $H$ \\
\hline \multirow[t]{5}{*}{ Juni } & 24 & $12^{h} \cdot 6$ & $9^{m} \cdot 5$ & - & $3^{\prime}$ & - & $7 \mathrm{mo}_{0}$ \\
\hline & 26 & 13.2 & $9 \cdot 5$ & 一 & 3 & $5^{\prime}-12^{\prime}$ & 6.6 \\
\hline & 27 & 13.4 & 9.0 & - & 3 & $8-20$ & 6 \\
\hline & 28 & 13.4 & 9.7 & $8^{m} \cdot 5$ & 3 & $8-\cdots$ & 5.1 \\
\hline & 29 & 13.5 & $9 \cdot 5$ & 一 & 3 & $8 \ldots$ & 4.9 \\
\hline \multirow[t]{19}{*}{ Juli } & 2 & 13.5 & 9 & - & 4 & $12-30$ & 4.8 \\
\hline & 3 & 13.6 & $9 \cdot 3$ & - & 3.5 & $8-\cdots$ & $4 \cdot 7$ \\
\hline & 4 & 13.7 & 9 & - & 4 & $12-40$ & 4.6 \\
\hline & I I & 10.2 & 9 & 8 & 4 & $3^{\circ}(\mathbb{C})$ & 4 \\
\hline & 12 & 10.0 & 9 & - & 4 & 30 & 3.8 \\
\hline & 13 & 10.0 & 9.5 & - & 5 & $15-\cdots$ & $3 \cdot 7$ \\
\hline & 14 & 10.4 & 9.5 & - & 5 & $20-60$ & 3.7 \\
\hline & 15 & 10.0 & 9 & $>8$ & 5 & $30-60$ & $3 \cdot 3$ \\
\hline & 16 & 10.0 & 8.5 & 一 & 6 & - & 3.1 \\
\hline & 18 & 10.0 & - & - & - & - & 2.8 \\
\hline & 18 & $13 \cdot 5$ & 8 & 6 & $6-8$ & $>60$ & - \\
\hline & 19 & 9.8 & 一 & 一 & - & 一 & 3.0 \\
\hline & 19 & 13.2 & 8 & - & 8 & $>60$ & - \\
\hline & 22 & 9.7 & 8 & 一 & $8-10$ & $40-\cdots$ & 3.0 \\
\hline & 23 & 9.6 & - & - & - & - & 3.0 \\
\hline & 26 & 9.5 & 7.8 & - & $7^{-8}$ & $40-\cdots$ & 3.4 \\
\hline & 28 & $9 \cdot 5$ & 8.2 & 6.2 & 8 & $1^{\circ}-2^{0}$ & 3.6 \\
\hline & 29 & 9.7 & $\vec{a}$ & - & 一 & $\overline{1}$ & $3 \cdot 5$ \\
\hline & 31 & 9.9 & 8 & $5^{m}-6^{m}$ & 7 & $20^{\prime}-\cdots$ & 3.6 \\
\hline \multirow[t]{8}{*}{ Aug. } & 2 & 9.8 & 7 & - & 6 & 40 & $4 \cdot 3$ \\
\hline & 4 & $9 \cdot 7$ & 7.5 & 一 & $5-6$ & $12-\cdots$ & $4.4(())$ \\
\hline & 8 & $9 \cdot 4$ & 6.7 & 一 & $4-5$ & 10 & 4 (C) \\
\hline & 9 & 9.1 & 6.7 & - & 4 & I $5-\cdots$ & $3.8(\circlearrowleft)$ \\
\hline & I I & 9.2 & 6.2 & - & 4 & 40 & 3.9 \\
\hline & 14 & 10.0 & 6 & - & 4 & 40 & $3 \cdot 7$ \\
\hline & 15 & 8.8 & 6 & - & $3-4$ & $30-\cdots$ & 3.8 \\
\hline & 16 & $9 \cdot 3$ & - & - & 3 & $3^{\circ}$ & $3 \cdot 7$ \\
\hline
\end{tabular}

Die Vergleichungen, durch welche die Zahlen $H$ entstanden sind, werden in den Annalen der Wiener Sternwarte mitgeteilt werden. Ich möchte aber doch schon hier einige anfuhren. - Am 16. Juli erschien der Komet für das bloße Auge kaum schwächer als $\delta$ Cygni $\left(3^{\mathrm{m}} \mathrm{r}\right)$ und auch nur wenig schwächer als $\beta$ Draconis $(3 \stackrel{\mathrm{m}}{\circ})$. - Am 18. Juli war er wesentlich auffallender als $\delta$ und $\zeta$ Draconis $\left(3 m^{m}\right)$, auch noch etwas auffallender als $\beta$ und $\eta$ Draconis $(2 \mathrm{~m} \cdot 9)$, aber wesentlich schwächer als $\gamma$ Draconis $(2 \mathrm{~m}, 5)$. - Am Iq. Juli war er auffallender als $\zeta$ Draconis, fast so wie $\eta$ Draconis. - Am 29. Juli war er samt dem ohne Fernrohr erkenn-
In den ersten Tagen und überhaupt, wenn der Komet nach Mitternacht beobachtet wurde, habe ich, wenn es die Luftverhältnisse erlaubten, auch noch den Zeitpunkt beob. achtet, bis zu welchem der Komet, d. h. hier seine hellste Partie, bei zunehmendem 'Tageslicht im 6 zöll. Refraktor gesehen werden konnte; diese Zeitpunkte sind hier unter , Ex. tinktion. zusammengestellt und daueben auch die Helligkeiten der Sterne $(* *)$ angegeben, die nahe gleichzeitig mit dem Kometen unsichtbar wurden.

\begin{tabular}{|c|c|c|c|}
\hline 1903 & \multicolumn{2}{|c|}{$\begin{array}{l}\text { Extinktion } \\
\text { M. } Z \text {. Wien }\end{array}$} & $* *$ \\
\hline Juin 24 & $14^{1}$ & $50^{m}$ & $9^{\mathrm{m}} \cdot 3$ \\
\hline 26 & 14 & $5^{6}$ & 9.4 \\
\hline 27 & 15 & $\mathbf{I}$ & $9 \cdot 3$ \\
\hline 28 & 15 & 2 & 9.2 \\
\hline 29 & 14 & $59(+?)$ & 9.5 (Wolkenschleier) \\
\hline Juli 2 & I 5 & 5 & 9 \\
\hline 3 & 15 & 9 & $9 \cdot 0-9 \cdot 3$ \\
\hline 4 & 15 & 10 & 9 (dunstig) \\
\hline 16 & 15 & $3^{r}$ & 9.5 \\
\hline 22 & 15 & 27 & 9.0 \\
\hline
\end{tabular}

Es sei darauf aufmerksam gemacht, daß sich der Komet bei den acht ersten dieser Extinktionen in der Nahe des Meridians und zwar anfangs östlich, rom 2. Juli an westlich und mit jedem Tage in größerer Höhe befand.

Über den Schweif und den Kopf des Kometen ist noch das folgende bemerkt worden.

Juni 24. Koma gegen Sudwest verlängert und ausge. breitet. - Juni 26. Der sehr breite Schweifansatz ist gegen dev Stern BD. -3.5316 gerichtet. - Juni 29. Der Schweif geht über den vom Kern $5^{\prime}$ abstehenden Stern BD. +0.4780 und ist gegen BD. -0.4263 gerichtet. - Juli 3. Koma auf der Schweifseite viel mehr entwickelt und heller als auf der Scheitelseite. Schweif in $8^{\prime}$ Abstand vom Kern fast 8' breit, dann aber immer unsicherer begrenzt.

In der zweiten Hälfte des Juli und zwar zuerst am 18. und 19. waren nicht nur die Rănder des Schweifes, sondern auch die Koma sehr unbestimmt begrenzt, sodals auch die Dimension des Kopfes nicht sicher anzugeben war. Die vom Schweif deutlich sichtbare Partie war gegen das Ende hin immer mehr schmal, sodaß der Schweif den Eindruck eines langen, spitzen Kegels machte. - Am 28. Juli zeigte die Koma an der Scheitelseite des Kopfes, in der Richtung gegen $\delta$ Ursae maj., ungefähr $135^{\circ}$ von der Schweifrichtung abstehend, eine schweifartige, sehr breite, aber diffuse Verlängerung. - Am 2. August war der Schweif gegen BD. $+50 \% 1862$ gerichtet. 
Am 16. August zeigte sich der Schweif in einer über. ! raschend großen Helligkeit und in einer veuen Form. Es waren zwei Schweife zu sehen; der längere und hellere, in RA. vorangehende, gegen $\mathrm{BD} .+399^{\circ} 400$ (49 Ursae maj.) gerichtet und auch fast so weit zu sehen (3 Grad), der kürzere und schwächere gegen eine Stelle, an der sich die kleinen Sterne BD. $+37^{\circ}: 2147$ und 2150 befinden. Am 20. August schien diese Zweiteilung, nachdem der Komet inzwischen wegen ungüntiger Witterung nicht zu sehen ge. wesen war, noch vorhanden zu sein; weiter aber konnte sie

Wien, 1903 August 27. nicht mehr verfolgt werden, weil der Komet schon so tief gegen den Horizont rückte, daß vom Schweif nur die hellste Partie und $z$ war am 22 . August bis 24 einer Lănge von $5^{\circ}$ zu erkennen war. Überhaupt machte sich der Komet in diesen letaten Tagen nur durch seine helle Kerogegend bemerkbar, welche am 23. August wie ein schöner »globular cluster von $1 / 2^{\prime}$ bis $34^{\prime}$ Durchmesser erschien. Als Hellig. keit derselben wurde durch Vergleichungen im Sucherfernrohr mit den Sternen im Kleinen Löwen $46(4 \div 0)$ und $37(4 \% 8)$ am 20. August $4: 0$, am $2 \mathrm{I} \cdot 4^{\mathrm{m}} \cdot 3$, am $22.4 \% 2$ gefunden.

Beobacbtungen des Kometen 1903 IV (1903 c) am Repsoldschen Heliometer.

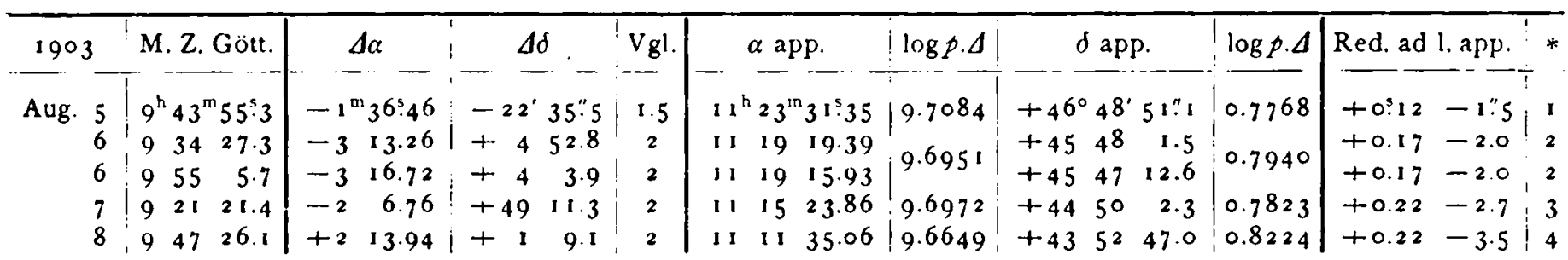

Mittlere Örter der Vergleichsterne.

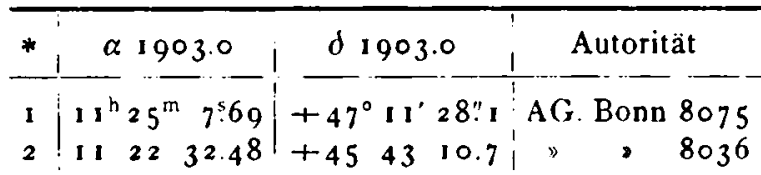

Göttingen, Sternwarte, 1903 Aug. 18.

\begin{tabular}{|c|c|c|c|}
\hline$*$ & $a 19 \circ 3.0$ & $\delta 1903.0$ & Autorität \\
\hline & 192 & $\begin{array}{l}-44^{\circ} 0^{\prime} \\
-4351\end{array}$ & \\
\hline
\end{tabular}

B. Meyermann.

\section{Beobachtungen des Kometen 1903 IV (1903 c)}

am r $3 \cdot z$ ölligen Refraktor der Sternwarte Königsberg.

(Fortsetzung zu A. N. 3890 ).

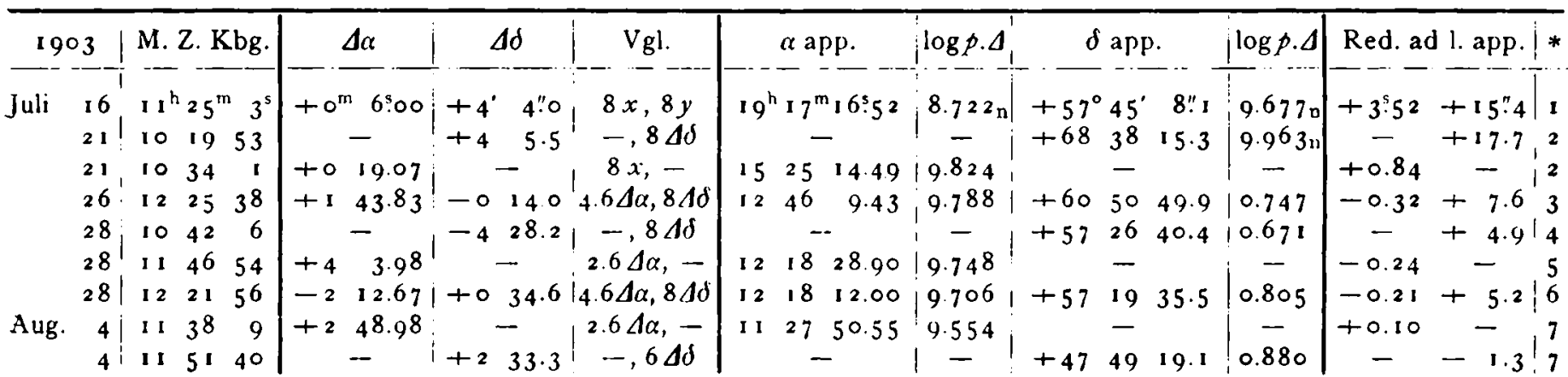

Beobachter: Juli 21, 28 I und II Prof. H. Struve; Juli 16, 26, 28 III, Aug. 4 A. Postelmann.

Mittlere Örter der Vergleichsterne.

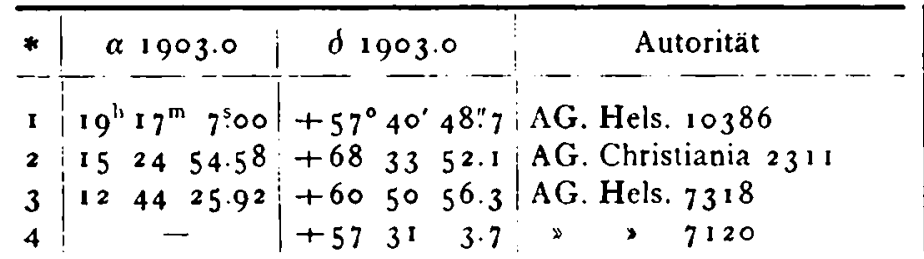

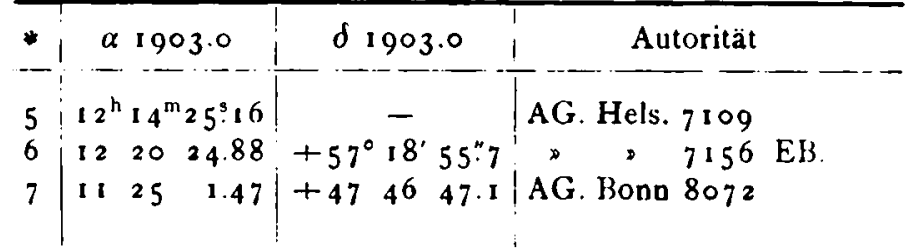

